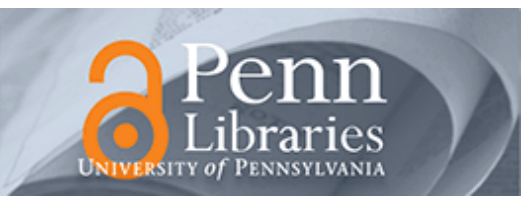

University of Pennsylvania

ScholarlyCommons

\title{
Toward tactilely transparent gloves: Collocated slip sensing and vibrotactile actuation
}

\author{
Joseph M. Romano \\ University of Pennsylvania, jrom@seas.upenn.edu \\ Steven R. Gray \\ University of Pennsylvania, stgray@seas.upenn.edu \\ Nathan T. Jacobs \\ University of Pennsylvania, njacobs@seas.upenn.edu \\ Katherine J. Kuchenbecker \\ University of Pennsylvania, kuchenbe@seas.upenn.edu
}

Follow this and additional works at: https://repository.upenn.edu/ese_papers

\section{Recommended Citation}

Joseph M. Romano, Steven R. Gray, Nathan T. Jacobs, and Katherine J. Kuchenbecker, "Toward tactilely transparent gloves: Collocated slip sensing and vibrotactile actuation", . April 2009.

Copyright YEAR 2009. Reprinted from:

Romano, J.M.; Gray, S.R.; Jacobs, N.T.; Kuchenbecker, K.J., "Toward tactilely transparent gloves: Collocated slip sensing and bibrotactile actuation," EuroHaptics conference, 2009 and Symposium on Haptic Interfaces for Virtual Environment and Teleoperator Systems. World Haptics 2009. Third Joint, vol., no., pp.279-284, 18-20 March 2009 URL: http://ieeexplore.ieee.org/stamp/stamp.jsp?arnumber=4810815\&isnumber=4810789

This material is posted here with permission of the IEEE. Such permission of the IEEE does not in any way imply IEEE endorsement of any of the University of Pennsylvania's products or services. Internal or personal use of this material is permitted. However, permission to reprint/republish this material for advertising or promotional purposes or for creating new collective works for resale or redistribution must be obtained from the IEEE by writing to pubs-permissions@ieee.org. By choosing to view this document, you agree to all provisions of the copyright laws protecting it.

This paper is posted at ScholarlyCommons. https://repository.upenn.edu/ese_papers/498

For more information, please contact repository@pobox.upenn.edu. 


\title{
Toward tactilely transparent gloves: Collocated slip sensing and vibrotactile actuation
}

\begin{abstract}
Tactile information plays a critical role in the human ability to manipulate objects with one's hands. Many environments require the use of protective gloves that diminish essential tactile feedback. Under these circumstances, seemingly simple tasks such as picking up an object can become very difficult. This paper introduces the SlipGlove, a novel device that uses an advanced sensing and actuation system to return this vital tactile information to the user. Our SlipGlove prototypes focus on providing tactile cues associated with slip between the glove and a contact surface. Relative motion is sensed using optical mouse sensors embedded in the glove's surface. This information is conveyed to the wearer via miniature vibration motors placed inside the glove against the wearer's skin. The collocation of slip sensing and tactile feedback creates a system that is natural and intuitive to use. We report results from a human subject study demonstrating that the SlipGlove allows the wearer to approach the capabilities of bare skin in detecting and reacting to fingertip slip. Users of the SlipGlove also had significantly faster and more consistent reaction to fingertip slip when compared to a traditional glove design. The SlipGlove technology allows us to enhance human perception when interacting with real environments and move toward the goal of a tactilely transparent glove.
\end{abstract}

\section{Keywords}

actuators, data gloves, sensors, SlipGlove, advanced actuation system, advanced sensing system, collocated slip sensing, human perception, miniature vibration motors, optical mouse sensors, protective gloves, tactile feedback, tactilely transparent gloves, vibrotactile actuation

\section{Comments}

Copyright YEAR 2009. Reprinted from:

Romano, J.M.; Gray, S.R.; Jacobs, N.T.; Kuchenbecker, K.J., "Toward tactilely transparent gloves: Collocated slip sensing and bibrotactile actuation," EuroHaptics conference, 2009 and Symposium on Haptic Interfaces for Virtual Environment and Teleoperator Systems. World Haptics 2009. Third Joint, vol., no., pp.279-284, 18-20 March 2009

URL: http://ieeexplore.ieee.org/stamp/stamp.jsp?arnumber=4810815\&isnumber=4810789

This material is posted here with permission of the IEEE. Such permission of the IEEE does not in any way imply IEEE endorsement of any of the University of Pennsylvania's products or services. Internal or personal use of this material is permitted. However, permission to reprint/republish this material for advertising or promotional purposes or for creating new collective works for resale or redistribution must be obtained from the IEEE by writing to pubs-permissions@ieee.org. By choosing to view this document, you agree to all provisions of the copyright laws protecting it. 


\title{
Toward Tactilely Transparent Gloves: Collocated Slip Sensing and Vibrotactile Actuation
}

\author{
Joseph M. Romano, Steven R. Gray, Nathan T. Jacobs and Katherine J. Kuchenbecker \\ Haptics Group, GRASP Laboratory \\ University of Pennsylvania, USA \\ \{jrom, stgray, njacobs, kuchenbe\}@seas.upenn.edu
}

\begin{abstract}
Tactile information plays a critical role in the human ability to manipulate objects with one's hands. Many environments require the use of protective gloves that diminish essential tactile feedback. Under these circumstances, seemingly simple tasks such as picking up an object can become very difficult. This paper introduces the SlipGlove, a novel device that uses an advanced sensing and actuation system to return this vital tactile information to the user. Our SlipGlove prototypes focus on providing tactile cues associated with slip between the glove and a contact surface. Relative motion is sensed using optical mouse sensors embedded in the glove's surface. This information is conveyed to the wearer via miniature vibration motors placed inside the glove against the wearer's skin. The collocation of slip sensing and tactile feedback creates a system that is natural and intuitive to use. We report results from a human subject study demonstrating that the SlipGlove allows the wearer to approach the capabilities of bare skin in detecting and reacting to fingertip slip. Users of the SlipGlove also had significantly faster and more consistent reaction to fingertip slip when compared to a traditional glove design. The SlipGlove technology allows us to enhance human perception when interacting with real environments and move toward the goal of a tactilely transparent glove.
\end{abstract}

\section{INTRODUCTION}

Humans use an array of sensory systems and mechanisms when manipulating objects by hand [7, 17]. Visual cues give insight into object properties, especially size, shape, and weight, which in turn enable an initial guess as to the level and placement of force necessary to properly handle the object $[10,11]$. Once contact is made, the somatosensory cortex responds to tactile information generated at the object-fingertip interface in order to optimize forces and achieve precise and accurate manipulation [20]. While several tactile inputs are utilized in this process, local micro-slips have been shown to play an essential role in grasp regulation [19].

In the absence of sufficient tactile feedback, people may mishandle an object during manipulation, risking injury to themselves and damage to the object. Even if the object is not technically mishandled, low levels of tactile input (originating from nerve injury, anesthesia, and glove use) have been associated with unnecessarily high grip forces at the object surface $[8,15]$. Overgripping may lead to discomfort, fatigue, and failure to complete essential tasks [3].

Thick gloves are often required to protect the hands from harm, but they also block important tactile information. Situations requiring this type of protection include environments dealing with extremes in pressure, such as space and undersea exploration [4]; extremes in temperature, such as freezers and ovens; and chemically hazardous situations, such as waste sites or chemical laboratories [2]. Though they keep the wearer's hands safe, standard

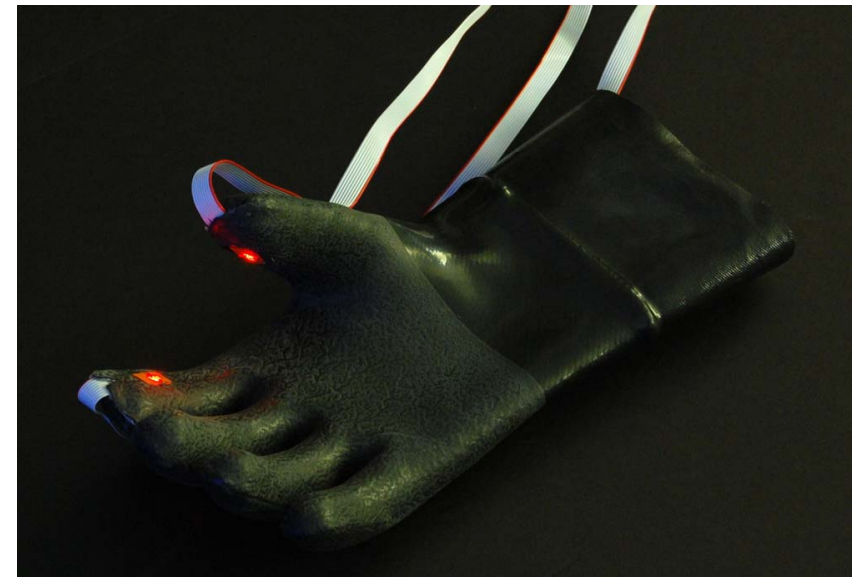

Figure 1: The SlipGlove V2. Note the presence of relative motion sensors on the inside of the thumb and index finger, with wires leading to an off-glove control box. When objects slide over the sensors, actuators inside the glove vibrate in response to the slip information.

protective gloves are far from tactilely transparent. Significant advances are required in the area of glove-based tactile feedback to give the wearer the sensory information that is available during bare-handed interactions. In order to address this issue, we have developed a system that provides thick-glove users with tactile feedback to compensate for the diminished tactile sensations experienced during glove use. While sensations of pressure, skin stretch, slip, and high-frequency vibration are all useful, we have focused on accurately detecting and displaying information related to slip at the glove surface. To restore this missing channel of information to users of thick gloves, we have created the SlipGlove (Fig. $1)$; the device incorporates collocated relative motion sensors and vibrotactile actuators to display slip information through pulses at the fingertips.

The ability of the glove to accurately alert the wearer to a slip event between the glove and an object was assessed in a human subjects study. In particular, it was hypothesized that the use of the SlipGlove would enable users to identify the occurrence of a slip event more quickly and consistently than they would when wearing a similar glove with no slip detection system.

\section{Previous Research}

To the authors' knowledge, the SlipGlove is the first system to collocate a relative motion sensor with a vibrotactile array to detect and alert users to slip events. While no prior published literature regarding this specific application has been found, the literature does contain studies in multiple fields related to the use of electromechanical systems to replace or augment tactile information in real, remote, and virtual contact situations.

Several researchers have created devices for the augmentation of 
human abilities in bare-hand contact scenarios. Kajimoto et al. [13] designed their SmartTouch system to act as a thin layer between the wearer's fingertips and the touched object. By using photosensors and electrocutaneous stimulation, they provide a fingertip interface to feel the difference between dark and light colored surfaces. Ando et al. [1] have developed a device mounted on the fingernail that uses a photodetector to sense the brightness of a surface and uses a voicecoil actuator to relay information to the user about the color and hue of the area being explored. In both of these designs the intended goal is to provide the wearer with a sixth sense about the visual appearance of the environment they are interacting with; notably, neither device attempts to alleviate the issue of sensory deprivation that the SlipGlove addresses.

A tactilely-enhanced glove was developed by Murray et al. [16] for use in teleoperation systems. These researchers augmented an Immersion CyberGlove using miniature voice coils arranged to form vibrotactile arrays on each of the fingertips of the system operator. On the remote end, a Utah-MIT artificial arm was equipped with fingertip-based force sensitive resistors capable of detecting forces experienced during object manipulation and transmitting the information back to the controller. The actuators on the operator's glove responded to this information with proportional vibrotactile feedback to allow the user to feel the forces being placed on the remote object. This system has several key differences to the SlipGlove in both the operating paradigm and mechanical systems employed. Most importantly, the SlipGlove focuses on transmitting slip information in real contact environments as opposed to representing force information from remote locations. However, multiple findings from the Murray study are applicable to the SlipGlove. Their study demonstrated that vibrotactile actuators were an effective tool for the relay of information to the human hand and that this form of feedback can stand in place of other tactile quantities, such as force or slip. They also found that proportional feedback was much more effective than more limited protocols, such as binary feedback. Other teleoperation and virtual environment systems with hand-based feedback include [9], [5].

Sensory substitution has also been occasionally applied to assistive technology [12], [18]. Cassinelli et al. recently showed that users wearing their Haptic Radar [6] system had an enhanced sense of spatial awareness. The Haptic Radar consists of a headband containing infrared proximity sensors connected to collocated vibration motors. As objects approach the head of the wearer, the modules on the headband begin to vibrate, giving cues about how close and from what direction an object is approaching. Human subjects were blindfolded and tested for their ability to avoid collisions with and without the Haptic Radar system. While the users were given minimal instruction on the principles of the Haptic Radar device, they almost immediately were able to use the feedback it provided to improve their performance in the task. Thus, with careful design choices such as the collocation of sensor/actuator pairs and simple proportional feedback relationships, haptic feedback can be used to enhance the capabilities of users in real environments. Our SlipGlove design emulates several of the principles that made the Haptic Radar an intuitive system for the end user, though we have focused on sensations that are naturally haptic, i.e. slip.

Lastly, Kikuuwe et al. [14] present a purely mechanical solution to the problem of sensory deprivation due to wearing gloves. By using the illusion that lateral skin stretch feels like normal deformation, the authors are able to create a 'tactile lens' layer that amplifies the effect of sliding one's hand over small surface discontinuities. As the user slides the tactile lens over a surface, they can feel an amplified version of the sheet's interactions with the surface below it, a sensation that is transmitted to the user's fingers via normal and tangential deformations of the sheet. A notable difference between this work and the SlipGlove is that the SlipGlove does not require the surface under inspection to have physical discontinuities

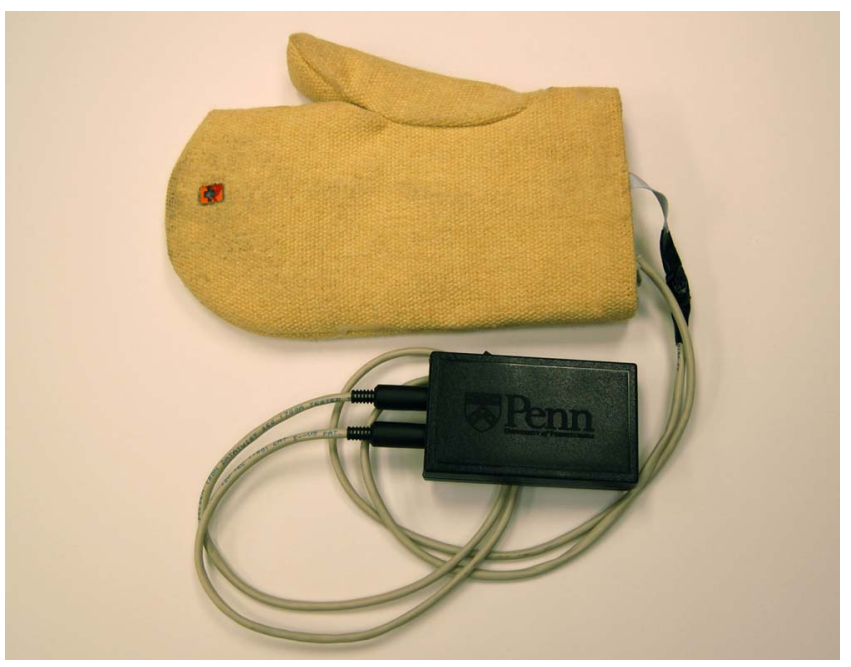

Figure 2: The SlipGlove V1. A single slip sensor has been placed in the area of the wearer's middle finger. The off-glove control box is wired to the glove via flexible cabling, and it can be clipped comfortably on the user's belt. This glove was used for all experiments described in this paper.

in order to transmit slip sensations back to the user. The findings by Kikuuwe et al. have not yet been applied to the problem of wearable gloves with multiple layers of differing material, but this approach may prove to be useful upon future research.

\section{Glove Design}

Two SlipGlove prototype devices have been constructed to date, versions one (V1) and two (V2), seen in Figures 2 and 1 respectively. V1 is a simplistic device created primarily for experimental purposes to evaluate human performance when using the glove technology. All of the experiments discussed in this paper were performed using the SlipGlove V1 system. V2 is a fully-functional prototype that extends the SlipGlove technology to a more complex multi-fingered domain.

The fundamental operating principles of the SlipGlove system are as follows: 1. Relative motion is detected by the sensors embedded in the fingertips of the glove. 2. An off-glove controller gathers information from the sensors and calculates the output signals to generate for the motors. 3. Output signals are sent to vibration motors embedded in the glove against the wearer's skin as a cue that

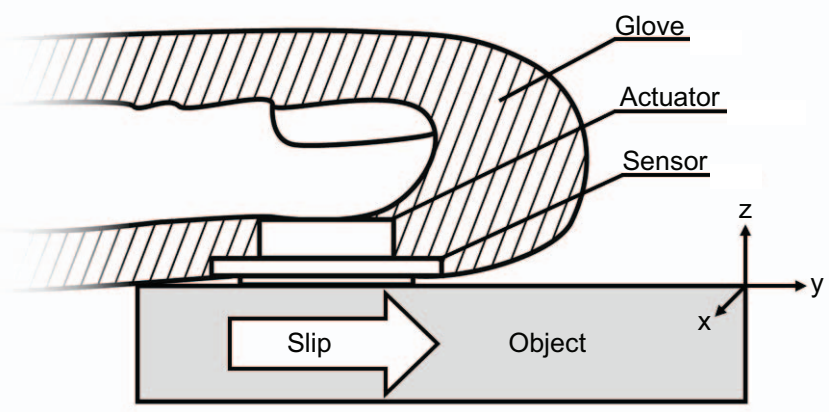

Figure 3: A cutaway depiction of the inside of the SlipGlove. As the object slips away from the finger in the y-direction, motion is detected by the sensor. The actuator, in contact with the finger, vibrates to inform the user of the slip event. 
a slip event is occurring. Special care was taken in designing an on-glove sensor/actuator suite that was miniaturized to be able to fit between the finger and the contact surface, while still being unobtrusive to the user. The placement of these components within the glove, as well as the basic slip detection task, is shown in Figure 3.

\subsection{Sensing}

The relative motion sensor used in our design is the ADNS-3530 from Avago Technologies. Commonly used in the PC industry as the sensing component in travel-sized optical mice, these sensors are desirable for their durability, reliability, energy efficiency, low cost, and small size. The surface-mount sensor package contains an onboard image acquisition system, surface illuminating LED, and digital signal processing hardware. The sensor uses this hardware to perform optical flow calculations, which result in planar relative motion estimates that are accurate at speeds up to 50.8 centimeters per second. An ADNS-3150-001 lens is used to focus our image acquisition system on surfaces within $1 \mathrm{~mm}$ of the sensor. The ADNS-3530 communicates with an off-glove controller over the SPI protocol at a bit rate of $1 \mathrm{MHz}$. Figure 4 shows an image of the sensor and its lens mounted on a custom fabricated printed circuit board (PCB). Figure 3 shows the planar degrees of freedom $(\mathrm{x}, \mathrm{y})$ for which the sensor returns relative motion information, and the depth direction $(\mathrm{z})$ in which the sensor can tolerate gaps of up to $1 \mathrm{~mm}$ to provide valid relative motion data.

Figures 1,2 and 3 all depict the mounting style of our relative motion sensors. The sensors are embedded in the glove such that they are flush with the glove's surface. Prototypes V1 and V2 differ in both the number of relative motion sensors and their placement on the glove. In V1 a single sensor is placed over what corresponds to the distal fingerpad of the middle finger. V2 has two sensors, one placed over the distal fingerpad of the index finger and one over the distal fingerpad of the thumb.

\subsection{Actuation}

The actuators used in our design are 312-101 (V1) and 310-101 (V2) shaftless vibration motors from Precision Microdrives Ltd (Fig. 4). These actuators were selected for their small size (12 mm and $10 \mathrm{~mm}$ diameter respectively), and because they are fully encased so that they can be placed in direct contact with the glove wearer without the possibility of the wearer interfering with the actuator's operation. Both motors are capable of creating vibrations with force amplitudes up to approximately $7.5 \mathrm{~N}$ at $175 \mathrm{~Hz}$. A linear relationship of $25 \mathrm{~Hz} / \mathrm{N}$ exists between the frequency and force amplitude. A mapping between input voltage and output frequency can be obtained as follows:

$$
\eta P_{e}=P_{m}
$$
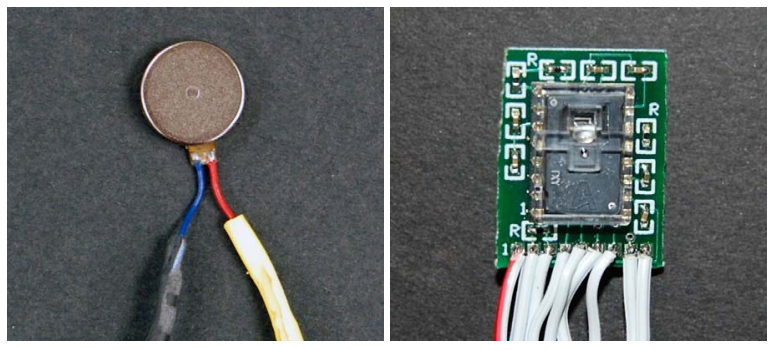

Figure 4: Left: The miniature vibration motor used inside the SlipGlove V1. Right: The relative motor sensor and lens used in the SlipGlove V1 and V2, mounted on a custom designed PCB. Both pictures have been scaled to the actual size of the objects.

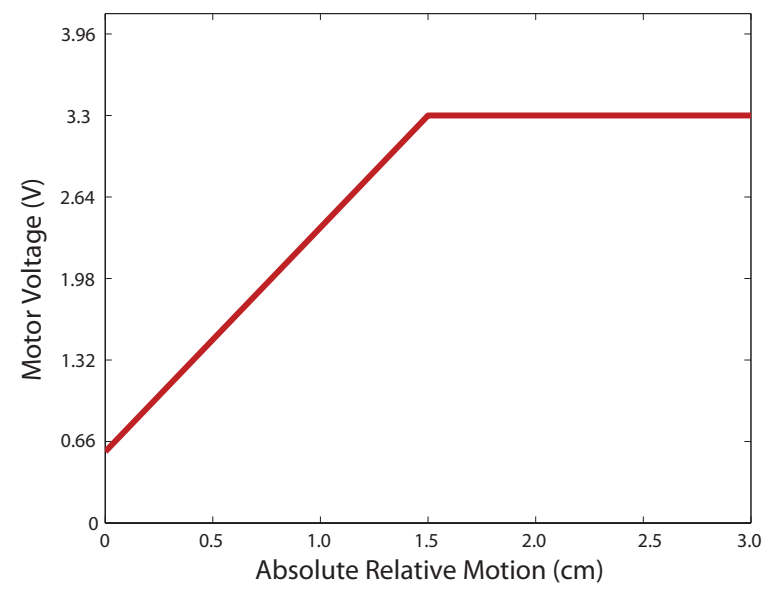

Figure 5: The mapping relationship between relative motion detected by the sensor and the output signal sent to SlipGlove V1's actuator. The output command was updated each time new relative motion was read from the sensor, at a rate of $20 \mathrm{kHz}(1 \mathrm{MHz} / \mathrm{bit})$. A voltage offset of $0.5775 \mathrm{~V}$ was necessary in order to overcome the initial motor deadband. A linear slope of $\frac{1.77 \mathrm{~V}}{\mathrm{~cm} / \mathrm{s}}$ was used until a cutoff point of $3.3 \mathrm{~V}$ was reached.

where $\eta$ is the motor efficiency, $P_{e}$ the input electrical energy, and $P_{m}$ the output mechanical energy. We can then substitute known relationships for the energy on both sides of the equation to obtain:

$$
\eta V i=\tau \omega
$$

where $V$ is voltage, $i$ is the current, $\tau$ is the motor torque, and $\omega$ the angular motor speed. Using the relationship for motor torque $\tau=k_{t} i$, where $k_{t}$ is the motor torque constant, we can rearrange the above equation to solve for motor velocity as:

$$
\omega=\frac{\eta V}{k_{t}}
$$

This result is valid for steady-state operation of the motor, assuming a sufficient current source is available. The constant value $\frac{\eta}{k_{t}}$ was calculated from manufacturer data to be about 60 revolutions/(V-s), providing a direct mapping from voltage to the oscillatory motion of our vibration motor. Experimentation with the actuators also showed that a deadband of $\pm 0.55 \mathrm{~V}$ exists before the motor begins to turn.

The actuators are mounted with industrial adhesive to the inside of the gloves, next to the wearer's skin (Fig. 3). A single 312101 vibration actuator is placed inside prototype V1, centered at approximately the same location as the slip sensor. A diamond pattern array of four 310-101 actuators is centered underneath each of the slip sensors in V2. An additional thin layer of foam cushioning exists between the actuator and the wearer's finger in V1 to enhance the comfort of the glove. V2 allows contact between the actuator's metal casing and the user's skin.

\subsection{Controller}

A custom control circuit reads data from the SlipGlove's relative motion sensors and generates output signals for its vibration actuators. This control circuit fits inside a small enclosure that is clipped on the user's belt (Fig. 3). At the center of the control circuit is an ATmega88 microcontroller. The ATmega88 reads data from the relative motion sensors via the SPI protocol at a rate of $20 \mathrm{kHz}$ ( $1 \mathrm{MHz} / \mathrm{bit})$. Output signals for the actuators are created by the ATmega88 in the form of $1 \mathrm{MHz}$ pulse width modulation (PWM) 
signals at an amplitude of 3.3 V. Before being sent to the vibration motors, these PWM signals are passed through a TCA0372 high current operational amplifier in the voltage follower (or buffer) configuration in order to obtain an adequate current supply for motor operation. The total delay time from the start of relative motion to the beginning of the motor output signal is 0.1 milliseconds for V1. The entire circuit, including the sensors and actuators, is powered by a single $9 \mathrm{~V}$ battery with a $3.3 \mathrm{~V}$ regulator.

Various mappings between sensor input and actuator array output are currently being explored in prototype V2. The mapping used in V1 for SlipGlove experiments discussed in this report is a proportional relationship between slip speed and actuator voltage. The euclidean distance that the glove has moved since the previous timestep is calculated in the code as: $D=\sqrt{d X^{2}+d Y^{2}}$, where $d X$ and $d Y$ represent the relative motion that has occurred since the last timestep in the two planar dimensions. The calculated value of $D$ is then used to determine the pulse width for our PWM output signal to the actuator, based on the linear relationship seen in Figure 5. The slope and offset of the Figure 5 graph were chosen such that relatively slow slip motions ( 0 to $1.5 \frac{\mathrm{cm}}{\mathrm{s}}$ ) fell within the linear regime of our output capabilities and were still detectable by the human user; this mapping was empirically validated to create a natural, salient feeling of slip when using the glove. Given the high $1 \mathrm{MHz}$ PWM frequency and the motor's tendency to act as a low-pass filter on the incoming PWM signal, we directly correlate the PWM duty cycle percentage $(0-100 \%)$ to an equivalent voltage level (0-3.3 V).

\section{USER EXPERIMENTS}

After designing the SlipGlove V1, we sought to determine its effect on the human ability to detect and react to slip events. An experiment was designed to test subject reaction time for three different conditions: the subject's bare hand, a normal glove, and the SlipGlove V1. The normal glove was a mitten identical to that used in the SlipGlove V1, without sensors or actuators. The material thickness of the SlipGlove V1 and normal glove was approximately $1 \mathrm{~cm}$. During the task, the user placed their hand atop a flat plate, which was then pulled away after a random time interval. The time it took the user to indicate that the plate was moving was recorded as the reaction time. By performing this controlled planar slip-detection task, we intended to gather data that would indicate whether the SlipGlove was useful in alerting the user to slip events during everyday tasks. We hypothesized that the SlipGlove would give users additional information about the slip event and thus allow them to react more quickly and consistently than when using the normal glove, and that these results should generalize to everyday slipping interactions. Such improvement should prove beneficial in grasping tasks.

\subsection{Test Apparatus}

The sliding plate that the users rested their hand upon was a 21.6 $\mathrm{cm} \times 19.0 \mathrm{~cm}$ piece of $0.635 \mathrm{~cm}$ thick acrylic (Fig. 6). Acrylic was chosen for its smooth finish, which causes it to easily slip underneath the fingertips. A wrist rest was constructed so that the user did not apply the entire weight of their arm to the sliding plate, but could be comfortable while performing the task. A custom designed linear actuator was used to slide the acrylic plate out from underneath the user's fingertips. The linear actuator consisted of a stepper motor attached to a belt driven car (Fig. 6), which was controlled through a PC via a LabJack U3 I/O interface and a UCN5804B stepper motor driver chip. By sending the stepper motor periodic step commands from the $\mathrm{PC}$, we were able to drive the system at a constant velocity of $2.03 \mathrm{~cm} / \mathrm{s}$. Each component of the system (the wrist rest, sliding plate, and linear actuator) was mounted to a separate surface to eliminate the transmission of vibration cues to the subject. In order to further prevent the stepper

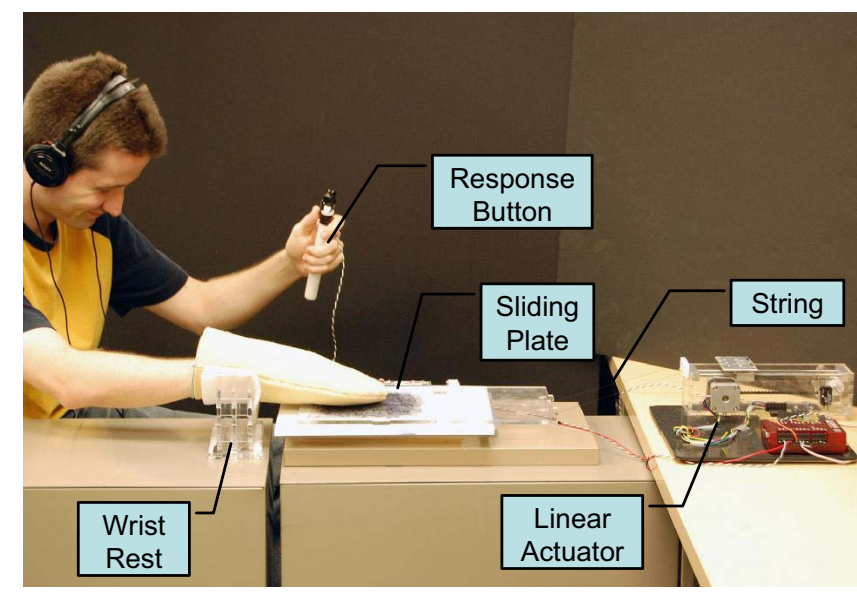

Figure 6: An example of a user performing a slip reaction trial on our test apparatus. Notice that the wrist rest, sliding plate, and linear actuator are all mounted to separate surfaces in order to minimize vibration transmission.

motor from vibrating the user's fingers, the linear actuator was connected to the sliding plate via a pair of strings.

To accurately detect the onset of plate movement, a magnet was placed on the sliding plate and a Hall effect sensor near the plate's starting point. The LabJack U3 was used to read in the voltage signal provided by the Hall effect sensor. When the voltage signal deviated by $0.1 \mathrm{~V}$ (a result of the distance between the sensor and plate increasing by approximately $1 \mathrm{~mm}$ ) the plate was considered to be moving. At this point a software timer was started to keep track of the time elapsed before the user clicked the response button (or the linear stage reached the end of its travel).

\subsection{Test Procedure}

A total of twelve subjects participated in the study. Eight of these were male and four were female. Only one user was left-handed, but he used his right hand to perform the trials. All subjects were between the ages of 22 and 30 . The experimental procedure for our human subject study is outlined below. All study procedures were approved by the Penn IRB, and subjects gave informed consent.

First, users were instructed to sit in a chair next to the test apparatus and hold the response button in their left hand. Next, one of the three test conditions (bare hand, normal glove, SlipGlove) was randomly selected by the experimenter. If the bare hand was selected, the user was given a small amount of Johnson's Baby Powder to rub on their right hand to unify the coefficient of friction between the hands of various users. If the normal glove or SlipGlove was selected, the user then put the selected glove on their right hand. The user was then instructed to place their wrist onto the wrist rest, and to rest their fingertips on the sliding plate. We informed the user that the plate would begin to slide out from underneath their fingertips at a random interval between one and five seconds after the start of the trial, and that they should click the response button as soon as they believed the plate was sliding. The user was also informed that they should apply a light amount of pressure on the object. Any trials where the user exerted enough pressure on the sliding plate to create a frictional force that overcame the pulling strength of the linear stage were discarded and repeated; this happened very rarely. A single trial was performed where the user was allowed to watch the test apparatus to gain an understanding of what was going to happen. The results of this trial were not recorded. The user then donned headphones playing a repeated pink-noise track to mask any audio information they might get from the system. The user was instructed to close their eyes until after they had clicked the re- 
sponse button or the experimenter had tapped them to notify them of a failed trial. The user's performance was recorded for eight subsequent trials in this manner, after which a different condition was selected and the entire process was repeated. Though they were recorded, the results of the first two trials were not analyzed to give subjects time to become familiar with the protocol. After eight trials for each of the three conditions, the user was asked to complete a short post-experiment questionnaire.

A trial was considered a failure when the user either pushed the response button too early (before the plate actually began to move), or never pushed the button at all. If the plate moved a distance of $10.2 \mathrm{~cm}$ without a response from the user, the trial was considered a failure and the experimenter informed the user that the trial had ended.

\section{Results}

The reaction times for successful trials of all users over the three tested conditions are represented in the histogram of Figure 7. For each user, we counted the number of failed trials that occurred in each condition and converted this to a proportion, $\rho_{\text {fail }}$. From the remaining successful trials, we calculated the mean $(\tau)$ and the standard deviation $(\varepsilon)$ for reaction time for this combination of user and condition (Table 1). One-sample repeated measures analysis of variance (ANOVA) by the multivariate approach was used to discern the trends in these three performance measures. The results of this analysis are reported below.

\subsection{Failure Rate}

As described in the test procedure above, trial failure was defined as whenever a user indicated that the plate was sliding too early or failed to detect sliding at all. The mean and standard deviation of the failure rate over all trials can be seen in Table 1. An ANOVA on failure rate between the three test conditions yielded: $(F(2,33)=28.6438, p=0.0001)$, where a $p$-value of 0.0001 shows that these differences are statistically significant (when compared with $\alpha=0.05$ ). Post-hoc pairwise t-tests resulted in p-values of 0.08821 , 0.00011, 0.00001, for the Bare Hand-SlipGlove, SlipGlove-Normal

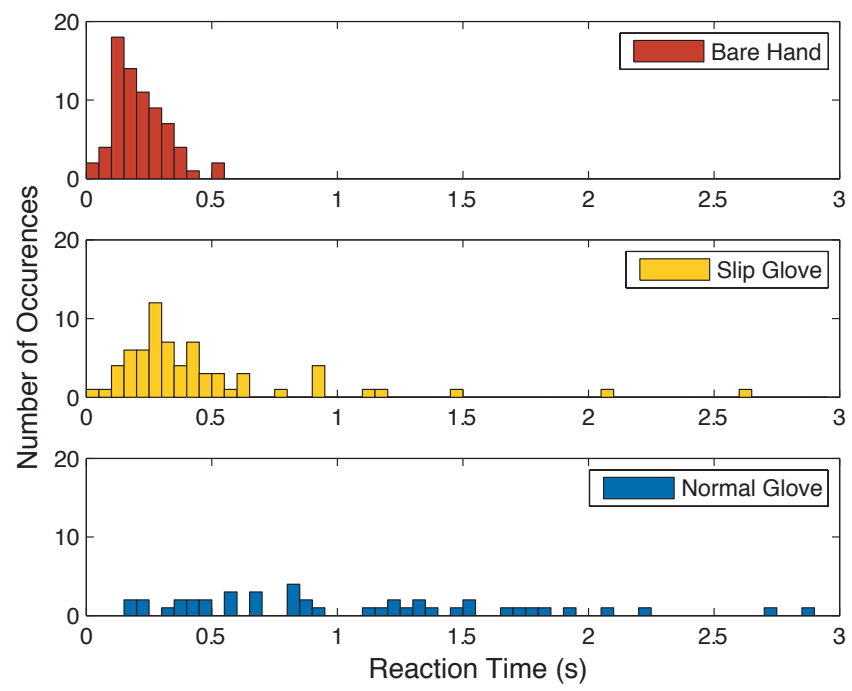

Figure 7: A histogram of all reaction time data collected in our human subject study. Bare hand data is clustered closely together in the region between 0 and $0.5 \mathrm{~s}$. SlipGlove data has a similar clustering at a slightly higher value, while normal glove data is spread widely throughout. In a post-trial survey many users reported not being able to feel any slip information while using the normal glove, and as a result they tried to guess when the plate was moving.

\begin{tabular}{|c|ccc|}
\hline & Bare Hand & SlipGlove & Normal Glove \\
\hline \multirow{2}{*}{$\rho_{\text {fail }}$} & $0.0 \%$ & $5.6 \%$ & $27.8 \%$ \\
& $(0.0 \%)$ & $(10.9 \%)$ & $(17.9 \%)$ \\
\hline \multirow{2}{*}{$\tau$} & $0.214 \mathrm{~s}$ & $0.483 \mathrm{~s}$ & $1.669 \mathrm{~s}$ \\
& $(0.088 \mathrm{~s})$ & $(0.313 \mathrm{~s})$ & $(1.29 \mathrm{~s})$ \\
\hline \multirow{2}{*}{$\varepsilon$} & $0.058 \mathrm{~s}$ & $0.288 \mathrm{~s}$ & $0.914 \mathrm{~s}$ \\
& $(0.031 \mathrm{~s})$ & $(0.251 \mathrm{~s})$ & $(0.550 \mathrm{~s})$ \\
\hline
\end{tabular}

Table 1: Experimental results for human reaction trails. $\rho_{\text {fail }}$ is the percent of failed trials, $\tau$ is the mean response time, and $\varepsilon$ is the mean standard deviation.

Glove, and Bare Hand-Glove comparisons respectively. This result shows that failure rates between the Bare Hand and SlipGlove conditions were not statistically different. However, the failure rate for the normal glove was higher than both the Bare Hand and SlipGlove by a statistically significant amount.

\subsection{Mean Reaction Time}

The mean and standard deviation of the reaction time can be seen in Table 1. An ANOVA on mean reaction time between the three test conditions yielded: $(\mathrm{F}(2,33)=15.3812, \mathrm{p}=0.00034)$, where a $\mathrm{p}$ value of 0.00034 shows that these differences are statistically significant. Post-hoc pairwise t-tests resulted in p-values of 0.00576 , $0.00149,0.00032$, for the Bare Hand-SlipGlove, SlipGlove-Normal Glove, and Bare Hand-Glove tests respectively. This result shows that all three conditions were statistically unique from one another Note that bare hand reaction time was the fastest, followed by the SlipGlove reaction time, with the normal glove being a distant third.

\subsection{Repeatability}

The mean of all subjects' reaction time standard deviations over all trials can be seen in Table 1. ANOVA tests on the reaction time standard deviation resulted in: $(\mathrm{F}(2,33)=22.7376, \mathrm{p}=0.0002)$, where a $\mathrm{p}$-value of 0.0002 shows that these differences are statistically significant. Post-hoc pairwise t-tests resulted in p-values of 0.00967 , 0.00593, 0.00017, for the Bare Hand-SlipGlove, SlipGlove-Normal Glove, and Bare Hand-Glove tests respectively. This result shows that the standard deviation in each condition was statistically unique. We note that the standard deviation of the bare hand was lowest, followed first by the SlipGlove, and finally by the normal glove. The bare hand reaction time task was more repeatable than the SlipGlove, which was more repeatable than the normal glove.

\section{Discussion And Conclusion}

The SlipGlove introduces a novel application of collocated relative motion sensors and vibrotactile actuators to restore the wearer's ability to detect and respond to slip between the glove-object interface. Prior work implied that this information was essential to object manipulation tasks, and the study presented in this paper confirms that users of the SlipGlove are better able to detect and respond to slip events compared to a normal glove. Overall, the SlipGlove managed to improve the mean reaction time of human subjects by 1.186 seconds (a $345 \%$ increase in performance). The SlipGlove has also been shown to dramatically improve the repeatability of slip detection and also reduce the failure rate for difficult tasks. We believe that as this technology is further developed, it will allow users of thick gloves to better interact with their environments, providing them with better manipulation abilities and allowing them to avoid overexertion and fatigue.

The technology presented in this paper excels in several areas. First, the collocation of the sensor and actuator on the fingertip provides an input to the user that requires no time to learn. Additionally, it is portable, as the controller mechanism can be worn on a 
belt, allowing the glove to be taken anywhere. Our V2 prototype glove was constructed for under $\$ 100$. The low price of the SlipGlove technology makes it affordable for integration into any existing glove. In a post-study survey users gave the SlipGlove high marks in terms of comfort (4.6/5.0) and usefulness (4.3/5.0) when compared to using the normal glove.

While the SlipGlove is a significant advancement in glove technology, further testing and development of this system will increase its effectiveness. We currently have plans to evaluate whether SlipGlove users demonstrate reduced grip forces when compared to users of normal gloves. Reducing the force applied by users when performing manipulation tasks will further highlight the usefulness of restoring slip information to the wearer. We also plan to improve the design of the device. In the current versions, the relative motion sensor is capped by a rigid cover, which does not flex around object surfaces. Depending on object surface qualities, this rigidity can make proper handling cumbersome. Once flexibility issues have been addressed, performance on grasping tasks will be evaluated experimentally. We hypothesize that some of the long reaction times seen in the middle panel of Figure 7 were due to inadvertent misalignment of the sensor with the surface. A flexible mount integrated into the glove would create better contact with objects.

The SlipGlove has experimentally been shown to allow users to approach reaction times similar to those of the bare hand. While further experimentation may continue to show improvement in a variety of other tasks, we are confident the SlipGlove provides a unique and exciting haptic augmentation for interaction with real environments.

\section{ACKNOWLEDGEMENTS}

We would like to thank Penn Haptics group member Will McMahan for lending his expertise and assistance in debugging the project's electronic hardware.

\section{REFERENCES}

[1] H. Ando, T. Miki, M. Inami, and T. Maeda. The nail-mounted tactile display for the behavior modeling. In International Conference on Computer Graphics and Interactive Techniques, pages 264-264, 2002.

[2] C. Bensel. The effects of various thicknesses of chemical protective gloves on manual dexterity. Ergonomics, 36(6):687-696, 1993.

[3] R. Bishu, V. Gnaneswaran, and A. Muralidhar. The Occupational Ergonomics Handbook. CRC Press, 1999.

[4] R. R. Bishu and G. Klute. The effects of extra vehicular activity (EVA) gloves on human performance. International Jounal of Industrial Ergonomics, 16:165-174, 1995.

[5] D. G. Caldwell, A. Wardle, O. Kocak, and M. Goodwin. Telepresence feedback and input systems for a twin armed mobile robot. IEEE Robitics \& Automation Magazine, September:29-38, 1996.

[6] A. Cassinelli, C. Reynolds, and M. Ishikawa. Augmenting spatial awareness with the haptic radar. In Proceedings of the Tenth International Symposium on Wearable Computers (ISWC), 2006.

[7] J. R. Flanagan, M. C. Bowman, and R. S. Johansson. Control strategies in object manipulation tasks. Current Opinion in Neurobiology, 16:650-659, 2006

[8] H. Groth and J. Lyman. Effects of surface friction on skilled performance with bare and gloved hands. Journal of Applied Psychology, 42(4):273-277, 1958.

[9] Immersion Corporation. Cybertouch technical specifications. http://www.immersion.com/3d/products/cybertouch.php. 2008.

[10] P. Jenmalm and R. S. Johansson. Visual and somatosensory information about object shape control manipulative fingertip forces. Jounal of Neuroscience, 17(11):4486-4499, 1997.

[11] R. S. Johansson, G. Westling, A. Backstrom, and J. R. Flanagan. Eyehand coordination in object manipulation. Journal of Neuroscience, 21(17):6917-6932, 2001.

[12] K. A. Kaczmarek, J. G. Webster, P. B. y Rita, and W. J. Tompkins. Electrotactile and vibrotactile displays for sensory substitution sys- tems. IEEE Transactions on Biomedical Engineering, 38(1):1-16, 1991.

[13] H. Kajimoto, N. Kawakami, S. Tachi, and M. Inami. Smarttouch: Electric skin to touch the untouchable. IEE Computer Graphics and Applications, 24:36-43, 2004.

[14] R. Kikuuwe, A. Sano, H. Mochiyama, N. Takesue, and H. Fujimoto. Enhancing haptic detection of surface undulation. ACM Transactions on Applied Perceptions, 2(1):46-67, January 2005.

[15] H. Kinoshita. Effect of gloves on prehensile forces during lifting and holding tasks. Ergonomics, 42:1372-1385, 1999.

[16] A. M. Murray, R. L. Klatzky, and P. K. Khosla. Psychophysical characterization and testbed validation of a wearable vibrotactile glove for telemanipulation. Presence, 12(2):156-181, 2003.

[17] D. A. Nowak, S. Flasauer, and J. Hermsdorfer. How predictive is grip force control in the complete abscence of somatosensory feedback? Brain, 127:1-11, 2004.

[18] S. Shoval, J. Borenstein, and Y. Koren. Auditory guidance with the navbelt - a computerized travel aid for the blind. IEEE Transactions on Systems, Man, and Cybernetics, 28(3):459-467, 1998.

[19] A. M. Smith. Some shear facts and pure friction related to roughness discrimination and the cutaneous control of grasping. Canadian Journal of Physiology and Pharmacology, 72:583-590, 1993.

[20] G. Westling and R. S. Johansson. Factors influencing the force control during precision grip. Experimental Brain Research, 53(2):277-284, 1983. 